\title{
THE MULTIPLICITY OF THE LYASHKO-LOOIJENGA MAPPING ON THE DISCRIMINANT STRATA OF EVEN AND ODD POLYNOMIALS
}

\author{
CLARE BAINES \\ Department of Mathematical Sciences, Pure Mathematics Division \\ University of Liverpool \\ Liverpool L69 3BX, UK \\ E-mail: cebaines@liverpool.ac.uk
}

Let $M$ be a compact connected Riemann surface and let $S^{2}$ be the Riemann sphere. We say that two meromorphic functions $f_{1}: M \rightarrow S^{2}, f_{2}: M \rightarrow S^{2}$ are topologically equivalent if there exists a homeomorphism $h: M \rightarrow M$ such that $f_{1}=f_{2} \circ h$. Topologically equivalent meromorphic functions have the same types of critical values.

Generalised Hurwitz Problem. Find the number of topologically distinct meromorphic functions for a given distribution of critical points on distinct critical levels.

Hurwitz reduced this to a combinatorial problem and conjectured the solution for rational functions with one degenerate critical level (proven combinatorially in 1996 by Goulden and Jackson [?] and Strehl [?], and from the singularity point of view in 1997 by Goryunov and Lando [?]).

Lando and Zvonkine [?] solved the problem for non-Morse polynomials with fixed degenerate critical levels and we obtain a solution for even and odd polynomials. Our main tool, as it is in [?], is the Lyashko-Looijenga, LL, mapping which associates to a polynomial of degree $n$ the unordered set of its $n-1$ critical values. In particular we calculate the number of topological types of even and odd polynomials in a given discriminant stratum.

The discriminant of a family of polynomials is the set of polynomials with multiple roots. Within this set lie various strata determined by the distribution of critical points on fixed critical levels. We call these strata discriminant strata and we define them using passports. The number of topological types that we are seeking is closely related to the degree of the $L L$ mapping on the discriminant strata.

1991 Mathematics Subject Classification: Primary 57R45; Secondary 05C30.

The paper is in final form and no version of it will be published elsewhere. 
The passports mentioned above also define certain types of graph called cacti. The famous theorem of Cayley states that there are $(n+1)^{n-2}$ cacti of degree $n$ with passport $\mathbf{X}=\left\{X_{1}, \ldots, X_{n}\right\}, X_{i}=1^{n-2} 2^{1}$. In [?], Looijenga suggested a new proof for the Cayley theorem based on a relation between polynomial coverings of the Riemann sphere and cacti. He showed that each polynomial determines a unique cactus (up to homeomorphism) and, according to Riemann's Existence Theorem, that the converse is also true. Thus the number of types of topologically equivalent polynomials on a stratum defined by a passport is related to the number of topologically distinct cacti with this passport.

This paper is organised as follows. In the first section we identify the link between cacti and complex polynomials. We also introduce a new method of representing a cactus called a modified cactus. In certain circumstances this simplifies the appearance of a cactus and thus aids in their classification. The second section defines a passport and explains how this represents a stratum in a family of polynomials. We also introduce some notations relating to these passports and give their geometrical interpretations. In Section 3 we specialise everything from the previous sections to the family of even polynomials. We then state the main theorem which calculates the number of topological types of even polynomials on fixed discriminant strata and prove it using the method employed by Lando and Zvonkine in [?]. In the final section we calculate the multiplicity of the $L L$ mapping on strata in the family of odd polynomials by using the main theorem of the previous section.

Acknowledgments. The author would like to thank V. V. Goryunov for formulating the problem and for his help in the preparation of this paper. Special thanks to S. Lando for useful discussion.

1. Cacti and complex polynomials. Let us consider a c-star which is a planar graph with $c$ black vertices labelled anti-clockwise by the integers $1, \ldots, c$ each joined by an edge to a white vertex as in Fig. ??.

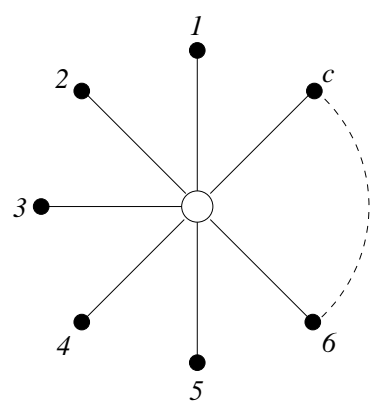

Figure 1. A $c$-star

Definition 1.1. A cactus of degree $n$ is a planar tree formed by gluing $n$ copies of a $c$-star in such a way that only black vertices of the same label are glued together. Such gluing ensures that all white vertices are of degree $c$, that is, there are $c$ edges meeting at the vertex, whereas the black vertices are of arbitrary degrees. 
Now let us number the white vertices $1, \ldots, n$. A natural permutation $g_{i} \in S_{n}$, $i=1, \ldots, c$, arises where $g_{i}$ acts on the white vertices surrounding the black vertex labelled $i$ by sending each white vertex anti-clockwise to the next one.

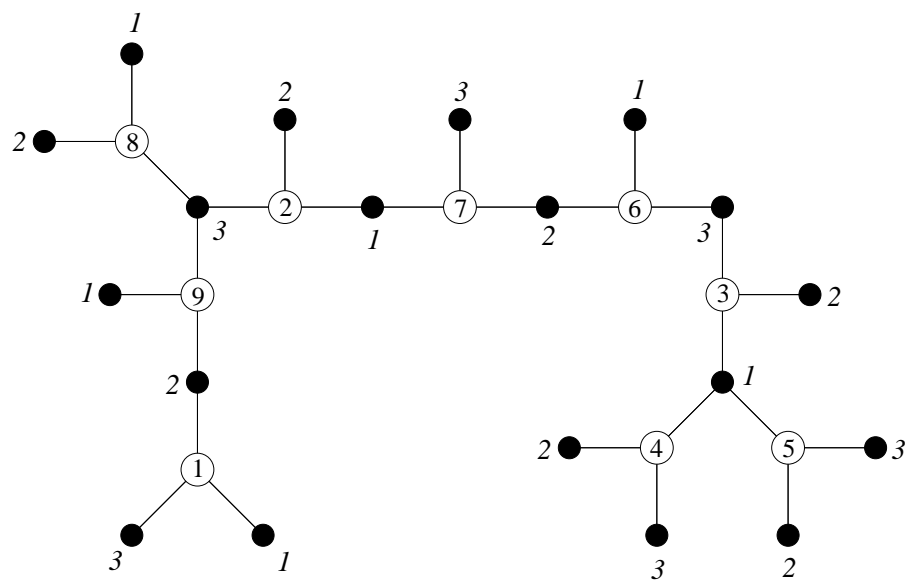

Figure 2. A cactus of degree 9

Example 1.2. Consider the cactus of degree 9 in Fig. ??. Here

$$
\begin{aligned}
& g_{1}=(2,7)(3,4,5), \\
& g_{2}=(6,7)(1,9), \\
& g_{3}=(2,8,9)(3,6) .
\end{aligned}
$$

Now consider the product $\gamma=g_{c} g_{c-1} g_{c-2} \cdots g_{1}$ which is an $n$-cycle, see [?]. By relabelling the white vertices we can fix $\gamma=(1,2, \ldots, n)$.

Definition 1.3. A rooted cactus of degree $n$, is a $c$-tuple of permutations

$$
C=\left[g_{1}, \ldots, g_{c}\right]
$$

on the set of integers $\{1, \ldots, n\}$ such that $\gamma=(1,2, \ldots, n)$. The root of the cactus is the white vertex number 1 .

ExAmPle 1.4. Using $g_{1}, g_{2}, g_{3}$ from Example ?? we can see that $\gamma=g_{3} g_{2} g_{1}=$ $(1,2,3,4,5,6,7,8,9)$. Thus the cactus in Fig. ?? is rooted.

Definition 1.5. Two rooted cacti $C_{1}=\left[g_{1}, \ldots, g_{c}\right]$ and $C_{2}=\left[h_{1}, \ldots, h_{c}\right]$ are isomorphic if there exists a permutation $\phi$ such that

$$
h_{1}=\phi^{-1} g_{1} \phi, \quad h_{2}=\phi^{-1} g_{2} \phi, \quad \ldots, \quad h_{c}=\phi^{-1} g_{c} \phi .
$$

As $\phi$ must preserve $\gamma$ we have $\phi=\gamma^{m}$, for some $m$. An equivalence class of a rooted cactus is called a non-rooted cactus.

Remark 1. It is clear that an asymmetric non-rooted cactus of degree $n$ produces $n$ different rooted ones. The only possible automorphism group of a non-rooted cactus is a cyclic group of order $s$, where $s$ divides $n$, and the group itself is generated by $\gamma^{m}$, $m=n / s$. Thus a symmetric non-rooted cactus produces $m=n / s$ different rooted cacti. 
To each permutation $g_{i}$, we assign a partition $X_{i}=1^{k_{(i, 0)}} 2^{k_{(i, 1)}} \cdots n^{k_{(i, n-1)}}$ of $n$ where the $k_{i, j-1}$ are the number of $j$-cycles in $g_{i}$.

Definition 1.6. The set $\left\{X_{1}, X_{2}, \ldots, X_{c}\right\}$ of partitions associated to the permutations $g_{i}$ is called the passport of the cactus. The set of all topologically distinct (up to homeomorphism) cacti with this passport is called the family of cacti.

Now we consider the relationship between complex polynomials and cacti. Let $P$ : $\mathbf{C P}^{1} \rightarrow \mathbf{C P}^{1}$ be a polynomial

$$
P(z)=z^{n}+\alpha_{1} z^{n-2}+\alpha_{2} z^{n-3}+\ldots+\alpha_{n-1} .
$$

with finite critical values $t_{1}, \ldots, t_{c}$. Let us choose an arbitrary non-critical value $t_{*} \in \mathbf{C}$. Join the point $t_{*}$ to all critical values by smooth non-intersecting and non-selfintersecting paths such that the paths are numbered anti-clockwise (about $t_{*}$ ) from 1 to $c$ and the $i$ th path joins $t_{*}$ to $t_{i}$. This forms a $c$-star. The critical values $t_{1}, \ldots, t_{c}$ are the black vertices of the star and are labelled by numbers $1, \ldots, c$. The point $t_{*}$ is the white vertex.

The pre-image of a star under the polynomial mapping (??) forms a graph embedded in the source sphere $\mathbf{C P}^{1}$, whose vertices are the pre-images of the points $t_{*}$ and $t_{i}$, $i=1, \ldots, c$, and whose edges are the pre-images of the rays of the star. This graph is composed of $n$ stars glued together in some way at black vertices (the vertices are coloured in the same way as their images). Moreover, the black vertices can be labelled by the numbers $1, \ldots, c$ in the same way as their images. This graph is a tree.

Thus a polynomial $P$ determines a unique cactus (up to homeomorphisms of the paths). The following theorem, which is a particular case of Riemann's Existence Theorem, states the converse (see [?, ?]).

THEOREM 1.7. Let the following data be given

- a c-tuple of permutations $\left[g_{1}, \ldots, g_{c}\right], g_{i} \in S_{n}$, defining a cactus of degree $n$;

- arbitrary complex numbers $t_{1}, \ldots, t_{c}$.

Then there exists a polynomial $P(z)$ of the form (??) with $c$ critical values equal to $t_{1}, \ldots, t_{c}$, and with the corresponding cactus defined by $g_{1}, \ldots, g_{c}$. This polynomial is unique, up to an affine change of coordinates $z \mapsto a z, a \in \mathbf{C}, a=\sqrt[n]{1}$.

1.1. Modified cacti. In Section 3.4 we will see that when we take the preimage of a $c$-star under an even polynomial mapping we obtain a cactus with a natural symmetry. This natural symmetry makes it easy to recognise any cactus as being that of an even polynomial and thus to determine the topological types. However, in Section 4.4 we will see that this is not necessarily true for a cactus determined by an odd polynomial. In order to regain some sense of symmetry in this case, and thus enable us to recognise a cactus as being that of an odd polynomial, we need to make some slight modifications to the previous definition of a cactus.

Assume we have a polynomial $P$ of degree $n$ with critical values $t_{1}, \ldots, t_{c}$. Take $t_{\varepsilon}$ to be a non-critical value close to $t_{c}$. We form a $c$-star whose black vertices are the critical values $t_{1}, \ldots, t_{c}$ of $P$ and are labelled $1, \ldots, c$ and whose white vertex is the point $t_{\varepsilon}$ and 
labelled $\varepsilon$. The preimage of this $c$-star under $P$ is a cactus of degree $n$ as defined in the previous section. We now modify the star as follows: as $t_{\varepsilon}$ tends to $t_{c}$, the white vertex $\varepsilon$ tends towards the black vertex labelled $c$ to form a modified $c$-star with white vertex $c$, see Fig. ??. The preimage of a modified $c$-star under the mapping $P$ will be called a modified cactus of degree $n$.

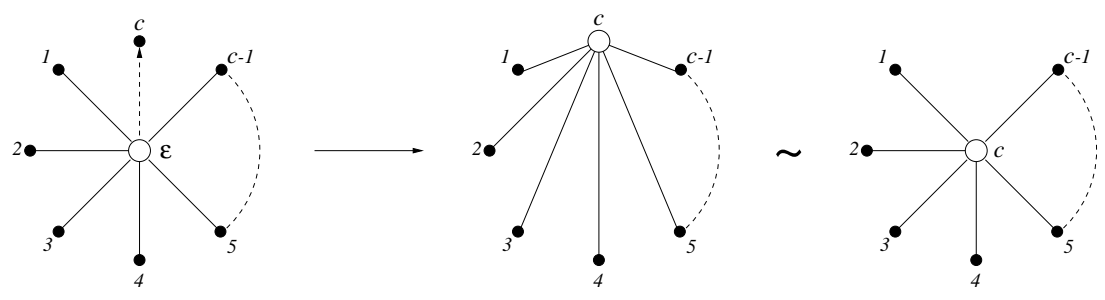

Figure 3. The new $c$-star

We can obtain a cactus which is topologically equivalent to this modified cactus by altering the original cactus in the following way. Assume that $P$ has a critical point of multiplicity $r$ with critical value $c$. Then the preimage of the original $c$-star under the mapping $P$ will contain $r$ copies of this $c$-star joined at a black vertex $c$. Now as $t_{\varepsilon}$ tends to $t_{c}$, the white vertices $\varepsilon$ tend towards the black vertex $c$. Thus the $r c$-stars join together to form a new white vertex (labelled $c$ in Fig. ??) surrounded by $r$ sets of black vertices which are labelled anti-clockwise $1, \ldots, c-1$; see Fig. ?? for the case when $r=2$.

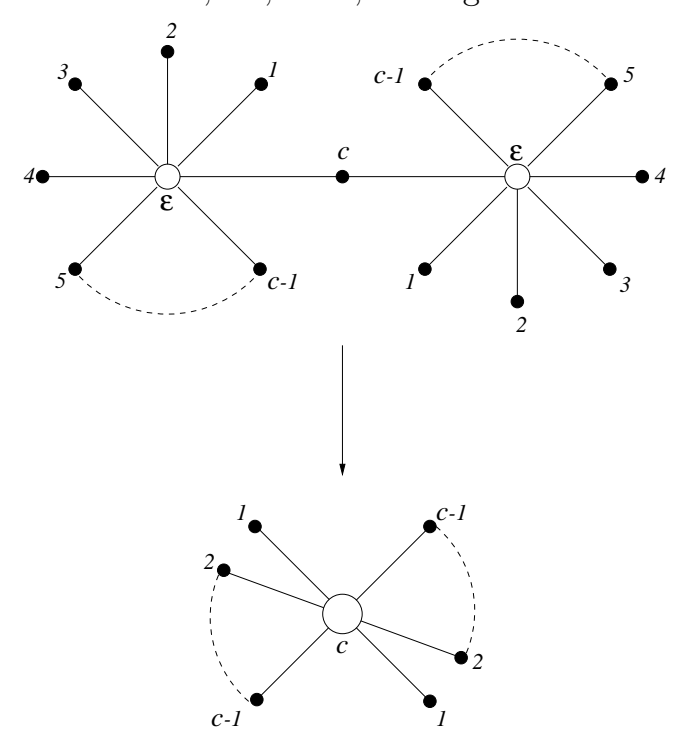

Figure 4.2 copies of a $c$-star joining together

Thus by modifying each of the copies of the $c$-star in the original cactus in this way we obtain a modified cactus with white vertices $c$.

Now consider the analogue of the permutations $g_{i}$ for ordinary cacti. Choose a base point $B$ close to the white vertex such that $B$ does not coincide with any of the black vertices. From this base point choose a counterclockwise loop $\beta_{c}$ around the white vertex 
and choose a counterclockwise simple loop $\beta_{i}, i=1, \ldots, c-1$, around each black vertex $i$. In this sense, simple means that each $\beta_{i}$ follows close to $\beta_{c}$ until it reaches the edge between the white vertex and the black vertex labelled $i$. The path then follows this edge. When it is close to vertex $i$ it moves anticlockwise around the black vertex. It then returns along the same path back to the base point. So we have simple paths $\beta_{i}$, $i=1, \ldots, c$, such that

1. each of the $\beta_{i}, i=1, \ldots, c$, has initial and end points $B$,

2. the $\beta_{i}$ do not intersect, for all $i$.
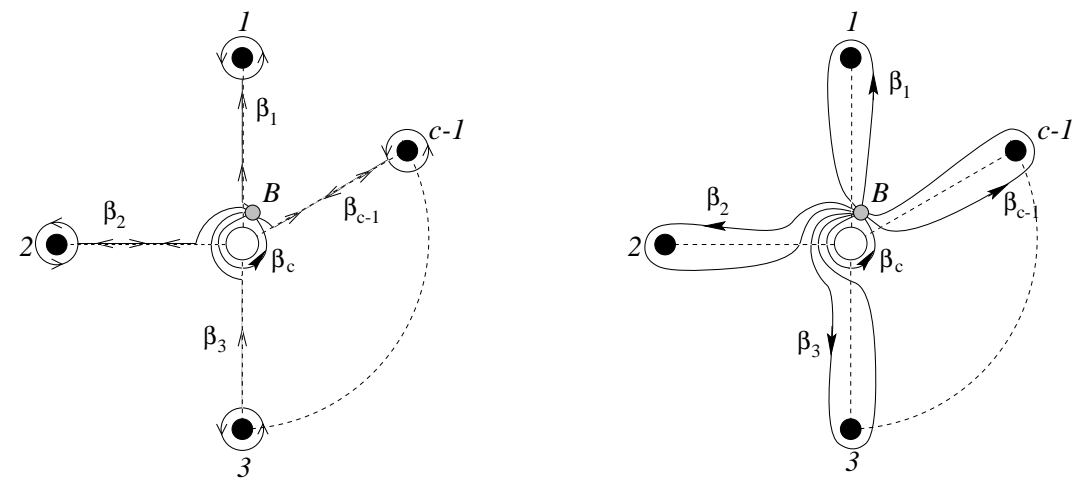

Figure 5. Example demonstrating how the loops may be chosen

For clarity, all diagrams will feature loops which are of the same homotopy type as the simple loops, rather than the simple loops themselves. Fig. ?? shows an example with simple loops on the left and, on the right, loops in the same homotopy class.
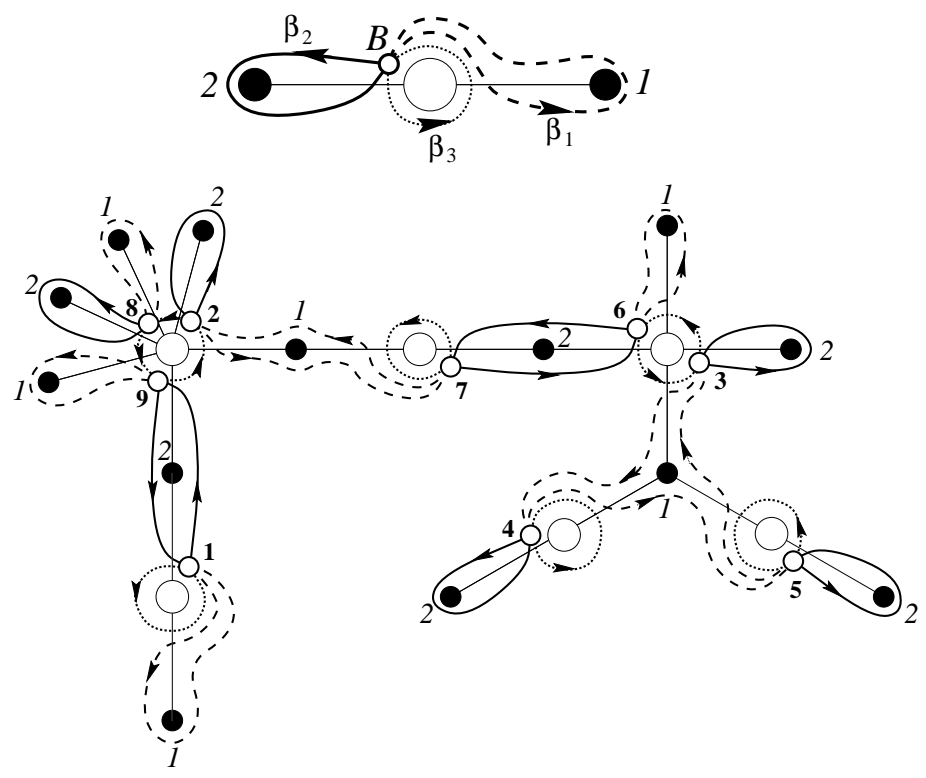

Figure 6. The modification of the cactus in Fig. 2 
Now take the preimage of these loops under the mapping $P$. We have $n$ copies of the base point $B$ which we label with the integers $\{1, \ldots, n\}$. The preimages of each of the $\beta_{i}$ form closed paths joining the preimages of the base point and passing around the preimages of the vertex $i$. See Fig. ??.

Let the permutation $b_{i}, i=1, \ldots, c$, act on the set of integers $\{1, \ldots, n\}$ in the following way: it sends a preimage of the base point to the next one along the preimages of the path $\beta_{i}$. Then $b_{i}=g_{i}$ and $b_{c} b_{c-1} \cdots b_{1}=\gamma$. Thus there is a one-to-one correspondence between an ordinary cactus and its related modified cactus.

Example 1.8. Consider the modification of the cactus in Example ??. Figure ?? shows the modified star with paths $\beta_{i}, i=1,2,3$, and the modified cactus with preimages of paths included. Then $b_{1}=(2,7)(3,4,5)=g_{1}, b_{2}=(1,9)(6,7)=g_{2}, b_{3}=$ $(2,8,9)(3,6)=g_{3}$.

2. Strata associated to a polynomial. Consider a polynomial $P$ of degree $n$ with a critical value $t$. There is a one-to-one correspondence between the multiplicities of the critical points of $P$ with this critical value and the roots of the equation $P(z)-t=0$. Assume that $P(z)-t=0$ has $k_{j}$ roots of multiplicity $j+1$, then the critical value $t$ of $P$ is attained at $k_{j}$ critical points of multiplicity $j, j=0, \ldots, n-1$, where $k_{0}=$ $n-\sum_{j=1}^{n-1}(j+1) k_{j}$. Using this, we can make the following definitions:

Definition 2.1. The partition of $n$ associated to $t$ is $X=1^{k_{0}} 2^{k_{1}} \cdots n^{k_{n-1}}$. We call a partition of this form the type of $X$.

Definition 2.2. The length of $X$ is $l(X)=k_{0}+k_{1}+\ldots+k_{n-1}$. This is the number of parts in $X$.

The automorphism group $\operatorname{Aut}(X)$ consists of permutations of the parts of $X$ such that $X$ retains its type. The order of this group is

$$
\# \operatorname{Aut}(X)=k_{0} ! k_{1} ! \cdots k_{n-1} ! \text {. }
$$

If $P$ has critical values $t_{1}, \ldots, t_{c}$ then each of these has an associated partition $X_{1}, \ldots, X_{c}$ respectively.

Definition 2.3. The set of all associated partitions of a polynomial is called the passport of $P$, which we denote $\mathbf{X}=\left\{X_{1}, \ldots, X_{c}\right\}$. This represents the degenerations of each of the critical values of $P$.

The automorphism group Aut $(\mathbf{X})$ consists of permutations of partitions $X_{i}$ of the same type, we denote its order by \#Aut(X).

Definition 2.4. To any passport $\mathbf{X}=\left\{X_{1}, \ldots, X_{c}\right\}$ we assign the stratum $\Sigma_{\mathbf{X}} \in$ $\mathbf{C}^{n-1}$. This stratum is the closure of the variety of all polynomials whose passport is $\mathbf{X}$.

Now let us consider the multiplicities of the critical values of $P$. 
Definition 2.5. The degeneracy of $X$ is $A(X)=0 \cdot k_{0}+1 \cdot k_{1}+\ldots+(n-1) \cdot k_{n-1}$. This is the multiplicity of the critical value, $t$.

Remark 2. Notice that $l(X)=k_{0}+k_{1}+\ldots+k_{n-1}$, and $k_{0}=n-\left(2 k_{1}+3 k_{2}+\right.$ $\left.\ldots+n k_{n-1}\right)$, so

$$
l(X)=n-k_{1}-2 k_{2}-\ldots-(n-1) k_{n-1}=n-A(X) .
$$

Consider the Lyashko-Looijenga mapping, LL: $\mathbf{C}^{n-1} \rightarrow \mathbf{C}^{n-1}, L L: P(z) \mapsto D(t)$, where the roots of the degree $n-1$ monic polynomial $D$ are the critical values of $P$ counted with their multiplicities. The multiplicities of the critical values of $P$, and hence the multiplicities of the roots of $D$, form a partition $T(\mathbf{X})$ of $n-1$.

Definition 2.6. To any passport $\mathbf{X}=\left\{X_{1}, \ldots, X_{c}\right\}$ we assign a partition $T(\mathbf{X})=$ $\left(A\left(X_{1}\right), \ldots, A\left(X_{c}\right)\right)=1^{m_{1}} 2^{m_{2}} \cdots(n-1)^{m_{n-1}}$.

We define the automorphism group $\operatorname{Aut}(T(\mathbf{X}))$ to consist of permutations of the roots of $D$ which have the same multiplicity. It has order

$$
\# \operatorname{Aut}(T(\mathbf{X}))=m_{1} ! m_{2} ! \cdots m_{n-1} ! \text {. }
$$

Definition 2.7. To any passport $\mathbf{X}$ we assign the stratum $\Phi_{T(\mathbf{X})}$ in the space $\mathbf{C}^{n-1}$ of all monic degree $n-1$ polynomials. This stratum is the variety of all polynomials whose root multiplicities form the partition $T(\mathbf{X})$.

Remark 3. Notice that a set of partitions $\mathbf{X}=\left\{X_{1}, \ldots, X_{c}\right\}$ is a passport of a polynomial $P$ iff $\sum_{i=1}^{c} A\left(X_{i}\right)=n-1$.

\section{Even polynomials}

Definition 3.1. A polynomial $P: \mathbf{C P}^{1} \rightarrow \mathbf{C P}^{1}$ is even if for any $z$

$$
P(z)=P(-z) \text {. }
$$

Explicitly we can write $P$ as a polynomial of the form

$$
P(z)=z^{2 M}+p_{1} z^{2(M-1)}+\ldots+p_{M-1} z^{2}+p_{M},
$$

where $p_{i} \in \mathbf{C}, i=1, \ldots, M$ and $p_{i}=\alpha_{2_{i}-1}$ in equation (??). We call the space of all polynomials of the form (??) the family of even polynomials, denoted $\mathcal{P}$, and we will be working within this family throughout this section.

A generic even polynomial of degree $2 M$ has $2 M-1$ distinct critical points $z_{1}=0$, $\pm z_{i}, i=2, \ldots, M$, and $M$ distinct critical values $P\left( \pm z_{i}\right)=t_{i}, i=1, \ldots, M$. The passport associated to a generic even polynomial is of the form

$$
\mathbf{X}=\left\{X_{1}, \ldots, X_{M}\right\}
$$

where $X_{1}=1^{2(M-1)} 2^{1}$ is the partition associated to the critical value $t_{1}$ and $X_{i}=$ $1^{2(M-2)} 2^{2}, i=2, \ldots, M$, are the partitions associated to the remaining critical values. 
Definition 3.2. We call a critical value even Morse if there are exactly 2 nondegenerate critical points on this level. The associated partition of an even Morse critical value is called an even Morse partition and is of the form $X=1^{2(M-2)} 2^{2}$.

In this section we study the restriction of the $L L$ mapping from the space of polynomials of degree $2 M$ of the form (??) to the subspace of even polynomials. The main aim is to calculate the multiplicity of the $L L$ mapping on a given stratum in the space of even polynomials which is determined by its passport. In order to do this we must first define a specific type of stratum called a primitive stratum.

3.1. Primitive strata in the family of even polynomials. We consider strata in the family of even polynomials.

DEFinition 3.3. A primitive stratum is a stratum which contains polynomials with only one degenerate critical value. The corresponding passport is called a primitive passport.

Note that when we consider a "degenerate" critical value, the degeneracy is with respect to the family of even polynomials.

Consider the stratum with passport $\mathbf{X}=\left\{X_{1}, \ldots, X_{c}\right\}$. This can be uniquely represented as the intersection of the primitive strata whose degenerate levels have partitions $X_{1}, \ldots, X_{c}$. Of course, if $X_{j}$ represents a non-degenerate level then this "primitive stratum" will just be a generic stratum. For even polynomials there are two types of primitive strata.

1. A stratum of type $\mathbf{P}_{1}$ where the degenerate level is at $t_{1} \neq P(0)$, see Figure ??.

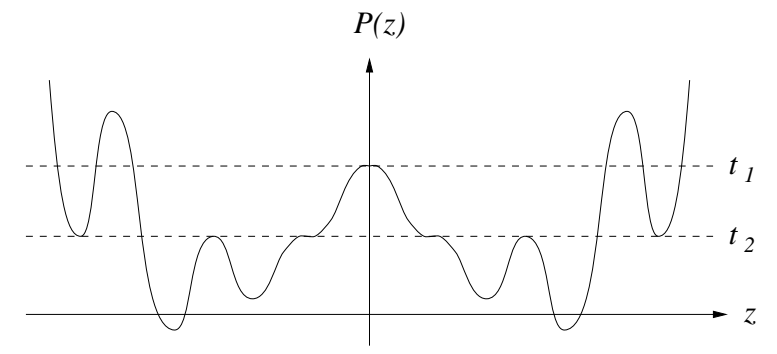

Figure 7. An example of a primitive stratum of type $\mathbf{P}_{1}$

The non-degenerate level $t_{2}=P(0)$ is attained at just one critical point $z=0$ and all other non-degenerate levels $t_{3}, \ldots, t_{c}$ are attained at 2 critical points each.

Let $X_{i}$ be the partition associated to the critical value $t_{i}, i=1, \ldots, c$.

A generic passport on the stratum $\mathbf{P}_{1}$ is

$$
\mathbf{X}_{\mathbf{P}_{1}}=\left\{X_{1}, X_{2}, X_{3}, \ldots, X_{c}\right\}
$$

where $X_{1}=1^{k_{0}} 2^{k_{1}} \cdots(2 M)^{k_{2 M-1}}, \quad k_{i}=2 \kappa_{i}$ for all $i$,

$X_{2}=1^{2(M-1)} 2^{1}$,

$X_{i}=1^{2(M-2)} 2^{2}, \quad i=3, \ldots, c$. 
Remark 4. Note, when considering primitive strata of type $\mathbf{P}_{1}$, that $k_{i}=0$ for $i>M-1$. However we include these $k_{i}$ in the above definition in order to retain some consistency in those definitions relating to both types of primitive strata which follow later on in this subsection.

2. A stratum of type $\mathbf{P}_{2}$ where the degenerate level is at $t_{1}=P(0)$, see Figure ??.

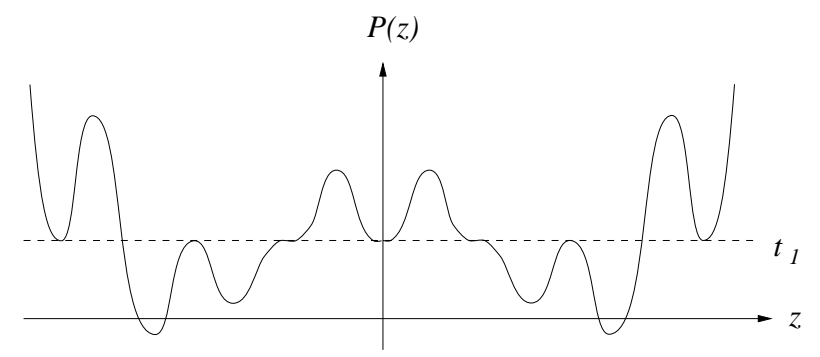

Figure 8. An example of a primitive stratum of type $\mathbf{P}_{2}$

The non-degenerate levels $t_{2}, \ldots, t_{c}$ are attained at 2 critical points each.

A generic passport on this stratum is

$$
\mathbf{X}_{\mathbf{P}_{2}}=\left\{X_{1}, X_{2}, \ldots, X_{c}\right\}
$$

where

$$
\begin{aligned}
& X_{1}=1^{k_{0}} 2^{k_{1}} \ldots(2 M)^{k_{2 M-1}}, \quad \begin{array}{l}
k_{2 j-1}=2 \kappa_{2 j-1}+1 \text { for one } j \in[2, \ldots, M] \\
k_{i}=2 \kappa_{i} \text { if } i \neq 2 j-1
\end{array} \\
& X_{i}=1^{2(M-2)} 2^{2}, \quad i=2, \ldots, c .
\end{aligned}
$$

We now introduce some notation. Recall, the definition of $A(X)$ as given in Def. ??.

Definition 3.4. To every primitive stratum, whose degenerate critical level $t_{1}$ has the associated partition $X_{1}$, we associate a partition $T\left(X_{1}\right)$ of $2 M-1$ as follows:

1. If we have primitive stratum of type $\mathbf{P}_{1}$ then

$$
T\left(X_{1}\right)=(A\left(X_{1}\right), 1, \underbrace{2, \ldots, 2}_{c-2})=12^{1} 2^{c-2} A\left(X_{1}\right)^{1}
$$

and $\# \operatorname{Aut}\left(T\left(X_{1}\right)\right)=(c-2) !$.

2. If we have primitive stratum of type $\mathbf{P}_{2}$ then

$$
T\left(X_{1}\right)=(A\left(X_{1}\right), \underbrace{2, \ldots, 2}_{c-1})=2^{c-1} A\left(X_{1}\right)^{1}
$$

and $\# \operatorname{Aut}\left(T\left(X_{1}\right)\right)=(c-1) !$.

Remark 5. Notice that if $X_{1}$ is even Morse then we have a generic stratum with $A\left(X_{1}\right)=2$ and $T\left(X_{1}\right)=(1, \underbrace{2, \ldots, 2}_{M-1})=1^{1} 2^{M-1}, \# \operatorname{Aut}\left(T\left(X_{1}\right)\right)=(M-1) !=(c-1) !$.

Definition 3.5. The sub-partition associated to a primitive stratum of type $\mathbf{P}_{1}$ or $\mathbf{P}_{2}$ is

$$
Y_{1}=1^{\kappa_{0}} 2^{\kappa_{1}} \cdots(2 M)^{\kappa_{2 M-1}} .
$$


Remark 6 . We have $l\left(Y_{1}\right)=\kappa_{0}+\ldots+\kappa_{2 M-1}=c-1$, where $c$ is the number of distinct critical values. We will now prove this.

1. If we have primitive stratum of type $\mathbf{P}_{1}$ then the multiplicity of the critical point with critical level $t_{1}$ is $A\left(X_{1}\right)$,

$t_{2}$ is 1 ,

$t_{i}$ is $2, \quad i=3, \ldots, c$.

Hence $A\left(X_{1}\right)+1+2(c-2)=2 M-1$. So $2 c-2=2 M-A\left(X_{1}\right)$. From Remark ?? we have that $2 M-A\left(X_{1}\right)=l\left(X_{1}\right)$. Thus $2 c-2=l\left(X_{1}\right)=2 l\left(Y_{1}\right)$.

2. If we have primitive stratum of type $\mathbf{P}_{2}$ then the multiplicity of the critical point with critical level $t_{1}$ is $A\left(X_{1}\right)$,

$t_{i}$ is $2, \quad i=2, \ldots, c$.

Hence $A\left(X_{1}\right)+2(c-1)=2 M-1$. So $2 c-1=2 M-A\left(X_{1}\right)=l\left(X_{1}\right)=2 l\left(Y_{1}\right)+1$.

\subsubsection{Multiplicity formulae for primitive strata}

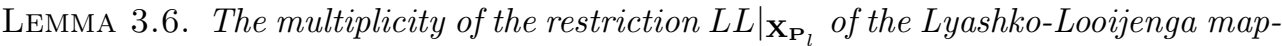
ping to the primitive strata of type $\mathbf{P}_{l}, l=1,2$, is given by the formula

$$
\mu_{\mathbf{X}_{\mathbf{P}_{l}}}=M^{c-1} \frac{\# \operatorname{Aut}\left(T\left(X_{1}\right)\right)}{\# \operatorname{Aut}\left(Y_{1}\right)}, \quad l=1,2 .
$$

Pro of. The mapping $\left.L L\right|_{\mathbf{X}_{\mathbf{P}_{1}}}$ defines a covering over the space of degree $M$ monic polynomials; this space is covered by the family of polynomials from the stratum of type $\mathbf{P}_{l}$. This mapping is finite, polynomial and quasihomogeneous (see [?]) and its multiplicity is obtained by applying the 'generalised Bézout theorem' (see [?]), defined as follows.

Theorem 3.7. Consider a finite quasi-homogeneous function $f: \mathbf{C}^{n} \rightarrow \mathbf{C}^{n}, f=$ $\left(f_{1}, \ldots, f_{n}\right)$ where each $f_{i}$ is of weight $w_{i}$, that is, for any $\lambda \in \mathbf{C}$,

$$
f_{i}\left(\lambda^{\beta_{1}} z_{1}, \ldots, \lambda^{\beta_{n}} z_{n}\right)=\lambda^{w_{i}} f\left(z_{1}, \ldots, z_{n}\right) .
$$

Then the multiplicity of $f, \mu(f)$, is given by the formula

$$
\mu(f)=\frac{w_{1} \cdots w_{n}}{\beta_{1} \cdots \beta_{n}} .
$$

We first consider the case when $l=1$. We can write a generic polynomial from $\mathbf{P}_{1}$ as

$$
P(z)=P_{0}(z) P_{1}^{2}(z) \cdots P_{2 M-1}^{2 M}(z)+t_{1}
$$

where $P_{i}(z)=z^{2 \kappa_{i}}+p_{i, 1} z^{2\left(\kappa_{i}-1\right)}+\ldots+p_{i, \kappa_{i}}, i=0, \ldots, 2 M-1$. Thus $\mathbf{P}_{1}$ is parametrised by the linear space of the coefficients of the $P_{i}$ and the critical value $t_{1}$.

The mapping $L L_{\mathbf{X}_{\mathbf{P}_{1}}}$ sends a polynomial (??) onto a polynomial $D=D(t)$ of degree $2 M-1$ whose roots are the critical values of $P$ counted with their multiplicities:

$$
D(t)=\left(t-t_{2}\right)\left(t-t_{1}\right)^{A\left(X_{1}\right)} D_{1}^{2}(t),
$$

where $D_{1}(t)=t^{c-2}+d_{1} t^{c-3}+\ldots+d_{c-2}$. Thus the image of $L L_{\mathbf{X}_{\mathbf{P}_{1}}}$ is parametrised by the linear space of the coefficients of $D_{1}(t)$ and the values $t_{1}, t_{2}$.

We now calculate $\mu_{\mathbf{X}_{\mathbf{P}_{1}}}$. Set the weight of $z$, denoted $\mathrm{wt}(z)$, be equal to 1 . Then the weights of the coefficients in the preimage of $L L$ are $\operatorname{wt}\left(p_{i, r}\right)=2 r, i=0, \ldots, 2 M-1$, 
$r=1, \ldots, \kappa_{i}$ and $\operatorname{wt}\left(t_{1}\right)=2 M$. The weights of the critical values of (??) $\operatorname{are} \operatorname{wt}(P(z))=$ $2 M$ hence $\operatorname{wt}(t)=\operatorname{wt}\left(t_{1}\right)=\operatorname{wt}\left(t_{2}\right)=2 M$, and $\operatorname{wt}\left(d_{i}\right)=2 M i, i=1, \ldots, c-2$. So by the generalised Bézout theorem

$$
\begin{aligned}
\mu_{\mathbf{X}_{\mathbf{P}_{1}}} & =\frac{\operatorname{wt}\left(t_{1}\right) \operatorname{wt}\left(t_{2}\right) \prod_{i=1}^{c-2}\left(d_{i}\right)}{\operatorname{wt}\left(t_{1}\right) \prod_{i=0}^{2 M-1} \prod_{r=1}^{\kappa_{i}} \operatorname{wt}\left(p_{i, r}\right)}=\frac{2 M \cdot 2 M \cdot(2 M)^{c-2}(c-2) !}{2 M \cdot 2^{\kappa_{0}+\ldots+\kappa_{2 M-1}} \cdot \kappa_{0} ! \cdots \kappa_{2 M-1} !} \\
& =\frac{(2 M)^{c} \# \operatorname{Aut}\left(T\left(X_{1}\right)\right)}{2 M \cdot 2^{l\left(Y_{1}\right)} \cdot \# \operatorname{Aut}\left(Y_{1}\right)}=\frac{(2 M)^{c}(c-2) !}{2 M \cdot 2^{c-1} \# \operatorname{Aut}\left(Y_{1}\right)}=M^{c-1} \frac{\# \operatorname{Aut}\left(T\left(X_{1}\right)\right)}{\# \operatorname{Aut}\left(Y_{1}\right)} .
\end{aligned}
$$

We now consider the case when $l=2$. We can write a generic polynomial from $\mathbf{P}_{2}$ as (4)

$$
P(z)=P_{0}(z) P_{1}^{2}(z) \cdots P_{2 M-1}^{2 M}(z)+t_{1}
$$

where

$$
P_{i}(z)= \begin{cases}z^{2 \kappa_{i}}+p_{i, 1} z^{2\left(\kappa_{i}-1\right)}+\ldots+p_{i, \kappa_{i}} & \text { for even } i \\ z\left(z^{2 \kappa_{i}}+p_{i, 1} z^{2\left(\kappa_{i}-1\right)}+\ldots+p_{i, \kappa_{i}}\right) & \text { for odd } i .\end{cases}
$$

The mapping $L L_{\mathbf{X}_{\mathbf{P}_{2}}}$ sends the polynomial (??) to a polynomial

$$
D(t)=\left(t-t_{1}\right)^{A\left(X_{1}\right)} D_{1}^{2}(t),
$$

where $D_{1}(t)=t^{c-1}+d_{1} t^{c-2}+\ldots+d_{c-1}$.

Now calculate $\mu_{\mathbf{X}_{\mathbf{P}_{2}}}$. Again take wt $(z)=1$. Then the weights of the coefficients in the preimage of $L L$ are $\operatorname{wt}\left(p_{i, r}\right)=2 r, i=0, \ldots, 2 M-1, r=1, \ldots, \kappa_{i}$ and $\operatorname{wt}\left(t_{1}\right)=2 M$, and the weights of the coefficients in the image of $L L$ are $\operatorname{wt}\left(t_{1}\right)=2 M$, and $\operatorname{wt}\left(d_{i}\right)=2 M i$, $i=1, \ldots, c-1$. So by the generalised Bézout theorem

$$
\mu_{\mathbf{X}_{\mathbf{P}_{2}}}=\frac{\operatorname{wt}\left(t_{1}\right) \prod_{i=1}^{c-1}\left(d_{i}\right)}{\operatorname{wt}\left(t_{1}\right) \prod_{i=0}^{2 M-1} \prod_{r=1}^{\kappa_{i}} \operatorname{wt}\left(p_{i, r}\right)}=M^{c-1} \frac{\# \operatorname{Aut}\left(T\left(X_{1}\right)\right)}{\# \operatorname{Aut}\left(Y_{1}\right)} .
$$

3.2. Main Theorem for even polynomials. Consider the passport $\mathbf{X}=\left\{X_{1}, \ldots, X_{c}\right\}$ with

$$
X_{j}=1^{k_{0, j}} 2^{k_{1, j}} \cdots(2 M)^{k_{2 M-1, j}}, \quad j=1, \ldots, c,
$$

where, for exactly one $j=\hat{\jmath} \in\{1, \ldots, c\}$ we have

$$
\begin{aligned}
k_{2 l-1, \hat{\jmath}} & =2 \kappa_{2 l-1, \hat{\jmath}}+1 & & \text { for exactly one } l \in\{1, \ldots, M\}, \\
k_{i, \hat{\jmath}} & =2 \kappa_{i, \hat{\jmath}} & & \text { if } i \neq 2 l-1,
\end{aligned}
$$

and otherwise

$$
k_{i, j}=2 \kappa_{i, j}, \quad \text { for each } i .
$$

To this passport we assign a partition

$$
T(\mathbf{X})=\left(A\left(X_{1}\right), \ldots, A\left(X_{c}\right)\right)=1^{m_{1}} 2^{m_{2}} \cdots(2 M-1)^{m_{2 M-1}},
$$

of $2 M-1$, where $m_{i}=\#\left\{X_{j} \in \mathbf{X}, A\left(X_{j}\right)=i\right\}$. Its automorphism group $\operatorname{Aut}(T(\mathbf{X}))$ contains

$$
\# \operatorname{Aut}(T(\mathbf{X}))=m_{1} ! \cdots m_{2 M-1} !
$$

elements. We denote by $T\left(X_{i}\right)$ and $Y_{i}$ the partition and sub-partition (respectively) associated to a primitive stratum with the degenerate critical level represented by $X_{i}$ (see Definitions ??, ??, respectively).

The number of even Morse partitions in the passport is denoted $c_{\text {Morse }}$. 
THEOREM 3.8. The multiplicity of the restriction $\left.L L\right|_{\mathbf{X}}$ of the $L L$ mapping to the stratum $\Sigma_{\mathbf{X}}$ determined by the passport $\mathbf{X}=\left\{X_{1}, \ldots, X_{c}\right\}$, is given by the formula

$$
\mu\left(\left.L L\right|_{\mathbf{X}}\right)=\frac{M^{c-1}}{(M-1)^{c_{\text {Morse }}}} \cdot \frac{\# \operatorname{Aut}(T(\mathbf{X}))}{\# \operatorname{Aut}(\mathbf{X})} \prod_{i=1}^{c} \frac{\# \operatorname{Aut}\left(T\left(X_{i}\right)\right)}{\# \operatorname{Aut}\left(Y_{i}\right)} .
$$

3.3. Proof of Main Theorem for even polynomials. Applying the methods used in [?] to our case we will now prove the Main Theorem.

Consider an even polynomial $P(z)$ of degree $2 M$ and the Lyashko-Looijenga mapping $L L: P(z) \rightarrow D(t)$ where the roots of the degree $2 M-1$ polynomial $D$ are the critical values of $P$ counted with their multiplicities. If $P$ is a generic even polynomial then $D$ is of the form $\left(t-d_{M}\right)\left(t^{M-1}+d_{1} t^{M-2}+\ldots+d_{M-1}\right)^{2}$ where $d_{M}=P(0)$.

Define $\mathcal{P}, \mathcal{D}$ to be the $M$-dimensional coordinate spaces of polynomials $P, D$ respectively (as defined above), and $\mathcal{A}, \mathcal{B}, \mathcal{T}$ to be the coordinate spaces $\left(a_{1}, \ldots, a_{M-1}, a\right)$, $\left(b_{1}, \ldots, b_{M}\right),\left(t_{1}, t_{2}, \ldots, t_{M}\right)$ respectively.

Consider the finite quasi-homogeneous mappings $\alpha: \mathcal{A} \rightarrow \mathcal{P}, \beta: \mathcal{D} \rightarrow \mathcal{B}$ and the Vièta mapping $\tau: \mathcal{T} \rightarrow \mathcal{D}$, where

$$
\begin{aligned}
& \alpha:\left(a_{1}, \ldots, a_{M-1}, a\right) \rightarrow P(z)=2 M \int_{0}^{z} \zeta\left(\zeta^{2}-a_{1}\right) \cdots\left(\zeta^{2}-a_{M-1}\right) d \zeta+a^{M}, \\
& \beta: D(t)=\left(t-d_{M}\right)\left(t^{M-1}+d_{1} t^{M-2}+\ldots+d_{M-1}\right)^{2} \rightarrow\left(b_{1}, \ldots, b_{M-1}, b_{M}\right), \\
& d_{M}=a^{M}, \quad b_{i}= \begin{cases}d_{i}^{(M-1) ! / i}, & i=1, \ldots, M-1 \\
d_{M}^{(M-1) !}, & i=M,\end{cases} \\
& \tau:\left(t_{1}, t_{2}, \ldots, t_{M}\right) \rightarrow\left(t-t_{1}\right)\left(\left(t-t_{2}\right) \cdots\left(t-t_{M}\right)\right)^{2} .
\end{aligned}
$$

Compose these mappings along with the Lyashko-Looijenga mapping $L L: \mathcal{P} \rightarrow \mathcal{D}$ as follows

$$
\begin{aligned}
\mathcal{A} \stackrel{\alpha}{\longrightarrow} \mathcal{P} \stackrel{L L}{\longrightarrow} & \mathcal{D} \stackrel{\beta}{\longrightarrow} \mathcal{B} \\
& \uparrow \tau \\
& \mathcal{T}
\end{aligned}
$$

Now we form the composite mappings

$$
F=\left(F_{1}, \ldots, F_{M}\right)=\beta \circ L L \circ \alpha: \mathcal{A} \rightarrow \mathcal{B}
$$

and

$$
G=\left(G_{1}, \ldots, G_{M}\right)=\beta \circ \tau: \mathcal{T} \rightarrow \mathcal{B}
$$

which are homogeneous of degrees $\operatorname{deg} F_{i}=M !$ and $\operatorname{deg} G_{i}=(M-1) !, i=1, \ldots, M$.

It is shown in [?] that if we consider a finite mapping $f: \mathbf{C}^{n} \rightarrow \mathbf{C}^{n}, f=\left(f_{1}, \ldots, f_{n}\right)$ such that each coordinate function $f_{i}$ is homogeneous of degree $w$, then we have

Lemma 3.9. Let $V \subset \mathbf{C}^{n}$ be a homogeneous variety of dimension $k$ in the source space such that $f(V)$ is irreducible. Then

$$
\operatorname{deg} V \cdot w^{k}=\operatorname{deg} f(V) \cdot \mu\left(\left.f\right|_{V}\right) .
$$

Here $\operatorname{deg} V, \operatorname{deg} f(V)$ are the degrees of the corresponding varieties, $\mu\left(\left.f\right|_{V}\right)$ is the multiplicity of the restriction of $f$ to the variety $V$. 
So we are now able to apply Lemma ?? to $F$ and $G$ since they are both homogeneous and finite. Consider a stratum $\Sigma \subset \mathcal{P}$ with $L L(\Sigma)=\Phi \subset \mathcal{D}$, and let $V \subset \mathcal{A}$ be a variety which covers $\Sigma$ via $\alpha$.

$$
\begin{array}{cccc}
\mathcal{A} \stackrel{\alpha}{\longrightarrow} \mathcal{P} \stackrel{L L}{\longrightarrow} \mathcal{D} \stackrel{\beta}{\longrightarrow} & \mathcal{B} \\
\cup & \cup & \cup & \cup \\
V \stackrel{\alpha}{\longrightarrow} \Sigma \stackrel{L L}{\longrightarrow} \Phi \stackrel{\beta}{\longrightarrow} \beta(\Phi)
\end{array}
$$

Since $V$ covers $\Sigma$, the dimension of $V$ is equal to the number of critical values of a generic polynomial in $\Sigma$, i.e. $\operatorname{dim} V=c$. Applying Lemma ?? to $F$ and $V$ we get

$$
\operatorname{deg} V \cdot(M !)^{c}=\operatorname{deg} \beta(\Phi) \cdot \mu\left(\left.F\right|_{V}\right)
$$

Also

$$
\mu\left(\left.F\right|_{V}\right)=\mu\left(\left.\alpha\right|_{V}\right) \cdot \mu\left(\left.L L\right|_{\Sigma}\right) \cdot \mu\left(\left.\beta\right|_{\Phi}\right)
$$

So

$$
\mu\left(\left.L L\right|_{\Sigma}\right)=(M !)^{c} \cdot \frac{\operatorname{deg} V}{\mu\left(\left.\alpha\right|_{V}\right)} \cdot \frac{1}{\mu\left(\left.\beta\right|_{\Phi}\right) \cdot \operatorname{deg} \beta(\Phi)} .
$$

So we now have to calculate the values of the components on the RHS of equation (??).

3.3.1. Strata in the space $\mathcal{D}$. We will begin by calculating the value of $\mu\left(\left.\beta\right|_{\Phi}\right) \cdot \operatorname{deg} \beta(\Phi)$ by applying Lemma ?? to $G$.

Lemma 3.10. Consider a stratum $\Phi=\Phi_{T(\mathbf{X})} \subset \mathcal{D}, T(\mathbf{X})=1^{m_{1}} \cdots(2 M-1)^{m_{2 M-1}}$. Then

$$
\mu\left(\left.\beta\right|_{\Phi}\right) \cdot \operatorname{deg} \beta(\Phi)=\frac{((M-1) !)^{c}}{\# \operatorname{Aut}(T(\mathbf{X}))}
$$

Pr o of. We prove this lemma by applying Lemma ?? to $G=\beta \circ \tau$. Choose the variety $W \subset \mathcal{T}$ to be one of the irreducible components of the preimage $\tau^{-1}(\Phi)$, i.e., an $M$-tuple such that the first $m_{1}$ coordinates $t_{j}$ are in general distinct, followed by $m_{2}$ pairs of coinciding numbers, followed by $m_{3}$ triplets of coinciding coordinates, etc.

So $\operatorname{deg} W=1, \operatorname{dim} W=m_{1}+\ldots+m_{2 M-1}=l(T)=c$, and $\operatorname{deg} G_{i}=(M-1) !$ Substituting these into Lemma ?? gives

$$
((M-1) !)^{c}=\operatorname{deg} G(W) \cdot \mu\left(\left.G\right|_{W}\right) .
$$

Now $\mu\left(\left.G\right|_{W}\right)=\mu\left(\left.\tau\right|_{W}\right) \cdot \mu\left(\left.\beta\right|_{\Phi}\right)$, and, since $\Phi$ is irreducible, $G(W)=\beta(\Phi)$. So we have

$$
((M-1) !)^{c}=\operatorname{deg} \beta(\Phi) \cdot \mu\left(\left.\tau\right|_{W}\right) \cdot \mu\left(\left.\beta\right|_{\Phi}\right) .
$$

We now calculate the degree of the covering $\left.\tau\right|_{W}$. There are $m_{i}$ ways of permuting blocks of length $i$ containing coinciding coordinates. Hence $\mu\left(\left.\tau\right|_{W}\right)=m_{1} ! \cdots m_{2 M-1}$ ! $=$ \#Aut $(T(\mathbf{X}))$. So we have

$$
\mu\left(\left.\beta\right|_{\Phi}\right) \cdot \operatorname{deg} \beta(\Phi)=\frac{(M-1) !^{c}}{\# \operatorname{Aut}(T(\mathbf{X}))} .
$$

3.3.2. Primitive strata in the space $\mathcal{P}$. Let us continue with the calculations of the values of the components on the RHS of equation (??). Since any stratum can be uniquely represented as the intersection of primitive strata, we will concentrate on finding values for the components in terms of primitive strata only. (The result in the previous section holds for any stratum.) 
LEMMA 3.11. Let $\Sigma \subset \mathcal{P}$ be a primitive stratum and let $V$ be an arbitrary union of irreducible components in the preimage $\alpha^{-1}(\Sigma) \subset \mathcal{A}$. Then

$$
\frac{\operatorname{deg} V}{\mu\left(\left.\alpha\right|_{V}\right)}=\frac{1}{M \# \operatorname{Aut}\left(Y_{1}\right)} \text {. }
$$

P r o of. Recall Lemmas ?? and ??. Substituting these into equation (??) with $\mathbf{X}=\mathbf{X}_{\mathbf{P}}$ gives

$$
\frac{\operatorname{deg} V}{\mu\left(\left.\alpha\right|_{V}\right)}=\frac{1}{(M !)^{c}} \cdot \frac{M^{c-1} \# \operatorname{Aut}\left(T\left(X_{1}\right)\right)}{\# \operatorname{Aut}\left(Y_{1}\right)} \cdot \frac{(M-1) !^{c}}{\# \operatorname{Aut}\left(T\left(X_{1}\right)\right)}=\frac{1}{M \# \operatorname{Aut}\left(Y_{1}\right)}
$$

We now need to choose a variety $V \subset \mathcal{A}$ so that we are able to compute the multiplicity $\mu\left(\left.\alpha\right|_{V}\right)$ and hence the degree of $V$.

Let $P$ be a generic polynomial from the primitive stratum $\Sigma_{\mathbf{P}} \subset \Sigma$ and suppose $t=t_{1}$ is its multiple critical value, with associated partition $X_{1}=1^{k_{0}} 2^{k_{1}} \cdots(2 M)^{k_{2 M-1}}$. Then $t_{1}$ is attained at $k_{i}$ critical points of multiplicity $i$, i.e., at $z=+\sqrt{a_{i}}$ and $z=-\sqrt{a_{i}}$, (where one of the $a_{i}$ may be zero). The total number of critical points on the degenerate level (when counted with multiplicity) is $1 k_{1}+2 k_{1}+\ldots+(2 M-1) k_{2 M-1}=A\left(X_{1}\right)$. Those critical points of $P$ which do not lie on the degenerate level are $z= \pm \sqrt{\widehat{a_{j}}}$, $j=A\left(Y_{1}\right)+1, \ldots, M-1$.

Define $V \subset \alpha^{-1}\left(\Sigma_{\mathbf{P}}\right) \subset \alpha^{-1}(\Sigma)$ to be an $M$-tuple which is determined as follows. The first $\kappa_{1}$ coordinates $a_{i}$ are distinct, followed by $\kappa_{2}$ pairs of identical coordinates, followed by $\kappa_{3}$ triplets of identical coordinates, etc. So we have $A\left(Y_{1}\right)$ coordinates of the form $a_{i}$ which relate to the critical points on the degenerate critical level of a generic polynomial from $\Sigma_{\mathbf{P}}$. The next (non-zero) $M-1-A\left(Y_{1}\right)$ coordinates are the $\widehat{a_{j}}$ as defined above, which are the squares of the critical points on the non-degenerate levels. The final coordinate is $a$ where $a^{M}$ is the degenerate critical level. We will call an $M$-tuple of this form a distinguished $M$-tuple, and the family of $M$-tuples a distinguished family of M-tuples.

Now calculate the degree of the covering $\left.\alpha\right|_{V}$. Consider $V$ as defined above. There are $\kappa_{i}$ ! ways of permuting the coordinates $a_{i}$ which relate to the critical points of multiplicity $i, 1<i<2 M-1$; there are \#Aut $\left(T\left(X_{1}\right)\right)$ ways of permuting those points $\widehat{a_{l}}$ which relate to the critical points which do not lie on the degenerate level; there are $M$ ways to choose an $a$ for a given value $a^{M}$. So

$$
\mu\left(\left.\alpha\right|_{V}\right)=M \cdot \kappa_{1} ! \cdots \kappa_{2 M-1} ! \cdot \# \operatorname{Aut}\left(T\left(X_{1}\right)\right) .
$$

Recall Lemma ??. Since \#Aut $\left(Y_{1}\right)=\kappa_{0} ! \kappa_{1} ! \cdots \kappa_{2 M-1}$ !, we have

$$
\operatorname{deg} V=\frac{\mu\left(\left.\alpha\right|_{V}\right)}{M \kappa_{0} ! \kappa_{1} ! \cdots \kappa_{2 M-1} !} .
$$

Substituting the value for $\mu\left(\left.\alpha\right|_{V}\right)$ attained above gives

$$
\operatorname{deg} V=\frac{\# \operatorname{Aut}\left(T\left(X_{1}\right)\right)}{\kappa_{0} !} .
$$


Remark 7. If $X_{1}$ is an even Morse partition, i.e., $X_{1}=1^{2(M-2)} 2^{2}$, then $\kappa_{0}$ ! $=$ $(M-2) !, A\left(X_{1}\right)=2$ and $\# \operatorname{Aut}\left(T\left(X_{1}\right)\right)=(M-1)$ !. So

$$
\operatorname{deg} V=(M-1) ! /(M-2) !=(M-1) .
$$

Notice that if $X_{1}=1^{2(M-1)} 2^{1}$, then $\operatorname{deg} V=1$.

We now recall equation (??)

$$
\mu\left(\left.L L\right|_{\Sigma}\right)=(M !)^{c} \cdot \frac{\operatorname{deg} V}{\mu\left(\left.\alpha\right|_{V}\right)} \cdot \frac{1}{\mu\left(\left.\beta\right|_{\Phi}\right) \cdot \operatorname{deg} \beta(\Phi)} .
$$

If $\Sigma$ is a primitive stratum we have values for each of the components of this equation.

3.3.3. Intersection of the primitive strata. To complete the proof of the main theorem for a stratum with passport $\mathbf{X}=\left\{X_{1}, \ldots, X_{c}\right\}$ we need to find values of each of the components of the RHS of equation (??) in terms of intersections of the primitive strata with degenerate levels represented by $X_{1}, \ldots, X_{c}$.

Let $c_{\text {Morse }}$ be the number of partitions of the form $X_{i}=1^{2(M-2)} 2^{2}$.

We choose in the pre-image $\alpha^{-1}\left(\Sigma_{X_{i}}\right)$ of each primitive stratum $\Sigma_{X_{i}}$ a union of irreducible components $V_{i}$ whose corresponding distinguished families of coordinates do not intersect. Let $V=\bigcap V_{i}$ be the intersection of all these components, then $\alpha(V)=\Sigma_{\mathbf{X}}$. The varieties $V_{i}$ intersect transversally at most points of $V$ so

$$
\operatorname{deg} V=\prod_{i} \operatorname{deg} V_{i}=\frac{1}{(M-1)^{c_{\text {Morse }}}} \prod_{1 \leq i \leq c} \frac{\# \operatorname{Aut}\left(T\left(X_{i}\right)\right)}{\kappa_{0_{i}} !} .
$$

Here $\kappa_{0_{i}}$ is the number of 1 's in the partition $Y_{i}$ and the factor $1 /(M-1)^{c_{\text {Morse }}}$ compensates for the contribution of even Morse partitions in the product which do not participate in the primitive strata intersections (see Remark ??).

Now

$$
\mu\left(\left.\alpha\right|_{V}\right)=M \# \operatorname{Aut}(\mathbf{X}) \prod_{1 \leq i \leq c} \frac{\# \operatorname{Aut}\left(Y_{i}\right)}{\kappa_{0_{i}} !}
$$

since there are \#Aut $(\mathbf{X})$ ways to permute the distinguished sets of $M$-tuples as wholes, \#Aut $\left(Y_{i}\right) / \kappa_{0_{i}}$ ! ways to permute $M$-tuples inside the $i$ th distinguished set and $M$ ways to choose $a$ for a given value $a^{M}$. So

$$
\frac{\operatorname{deg} V}{\mu\left(\left.\alpha\right|_{V}\right)}=\frac{1}{M(M-1)^{c_{\text {Morse }} \# \operatorname{Aut}(\mathbf{X})}} \prod_{1 \leq i \leq c} \frac{\# \operatorname{Aut}\left(T\left(X_{i}\right)\right)}{\# \operatorname{Aut}\left(Y_{i}\right)} .
$$

Substituting this and the expression for $\operatorname{deg} \beta(\Phi) \cdot \mu\left(\left.\beta\right|_{\Phi}\right)$ in equation (??) gives

$$
\mu\left(\left.L L\right|_{\mathbf{X}}\right)=\frac{M^{c-1}}{(M-1)^{c_{\text {Morse }}}} \cdot \frac{\# \operatorname{Aut}(T(\mathbf{X}))}{\# \operatorname{Aut}(\mathbf{X})} \prod_{i=1}^{c} \frac{\# \operatorname{Aut}\left(T\left(X_{i}\right)\right)}{\# \operatorname{Aut}\left(Y_{i}\right)}
$$

which completes the proof of Theorem ??.

3.4. Even cacti and even polynomials. An even cactus of degree $2 M$ is a centrallysymmetric cactus of degree $2 M$ whose passport $\mathbf{X}=\left\{X_{1}, \ldots, X_{c}\right\}$, is one associated to an even polynomial. 
We state the following theorem without proof. The result follows directly from the Goulden and Jackson theorem on the enumeration of cacti from [?] along with symmetry arguments.

Theorem 3.12. The number of rooted even cacti with passport $\mathbf{X}=\left\{X_{1}, \ldots, X_{c}\right\}$ is

$$
\frac{M^{c-1}}{(M-1)^{c_{\text {Morse }}}} \prod_{i=1}^{c} \frac{\# \operatorname{Aut}\left(T\left(X_{i}\right)\right)}{\# \operatorname{Aut}\left(Y_{i}\right)} .
$$

EXAMPle 3.13. Consider even cacti of degree 8 with passport

$$
\mathbf{X}=\left\{X_{1}, X_{2}, X_{3}\right\}=\left\{1^{6} 2^{1}, 1^{2} 3^{2}, 1^{4} 2^{2}\right\} .
$$

To this passport we associate the following values and partitions:

$M=4, c=3, c_{\text {Morse }}=1, T(\mathbf{X})=(1,4,2), T\left(X_{1}\right)=(1,2,2,2), T\left(X_{2}\right)=(4,1,2)$, $T\left(X_{3}\right)=(2,1,2,2), Y_{1}=1^{3}, Y_{2}=1^{1} 3^{1}, Y_{3}=1^{2} 2^{1}$.

Using the formula from Theorem ?? we calculate the number of rooted even cacti with passport $\mathbf{X}$ to be 16 . In fact the only possible topological types of cacti with this passport are those in Fig. ??. There are 4 rooted cacti of each topological type.

Now using the formula from Theorem ?? we calculate the multiplicity of the restriction of the $L L$ mapping to the stratum with passport $\mathbf{X}$ to also be 16 .
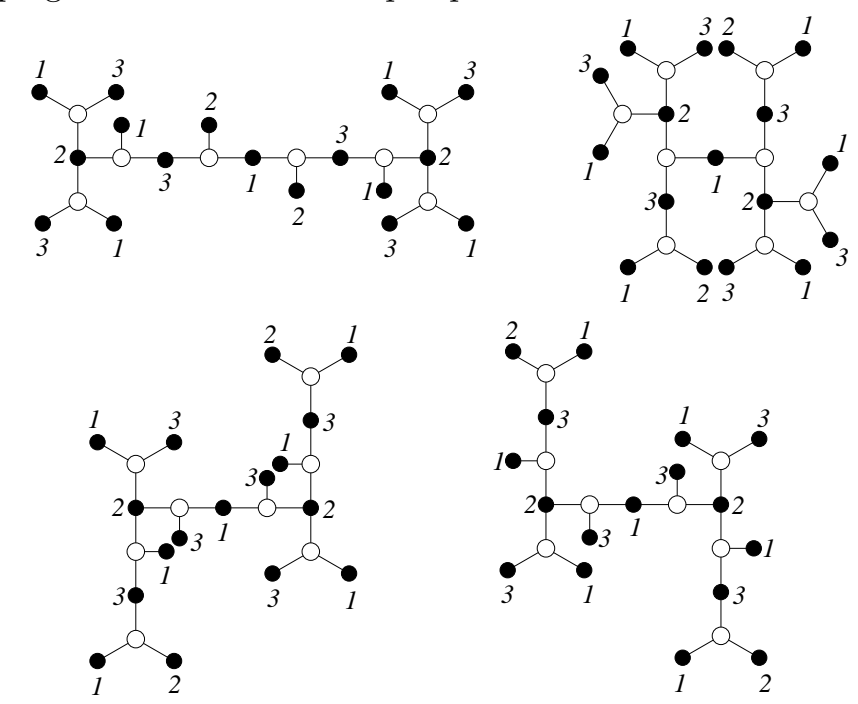

Figure 9. Unrooted even cacti with passport $\mathbf{X}=\left\{1^{6} 2^{1}, 1^{2} 3^{2}, 1^{4} 2^{2}\right\}$

\section{Odd polynomials}

Definition 4.1. A polynomial $P: \mathbf{C P}^{1} \rightarrow \mathbf{C P}^{1}$ is odd if for any $z$

$$
P(z)=-P(-z) \text {. }
$$

Explicitly we can write $P$ as a polynomial of the form

$$
P(z)=z^{2 M+1}+p_{1} z^{2 M-1}+p_{2} z^{2 M-3}+\ldots+p_{M} z
$$

where $p_{i} \in \mathbf{C}, i=1, \ldots, M$. 
4.1. Strata in the family of odd polynomials. We now determine the structure of a passport which defines a stratum in the family of odd polynomials. Throughout this section $P(z)$ is an odd polynomial of the form (??).

Let $t=t_{i}$ (and hence $\left.t=-t_{i}\right), i \geq 1$, be a non-zero critical value of $P$. Then $P(z) \pm t_{i}=0$ has $\kappa_{j, i}$ roots of multiplicity $j+1$, and hence each of the critical values $\pm t_{i}$ is attained at $\kappa_{j, i}$ critical points of multiplicity $j$. So to $t_{i}$ and $-t_{i}\left(=t_{-i}\right)$ we associate the partitions $W_{i}$ and $W_{-i}$ respectively where

$$
W_{i}=W_{-i}=1^{\kappa_{0, i}} 2^{\kappa_{1, i}} \cdots(M+1)^{\kappa_{M, i}},
$$

and $\kappa_{0, i}=2 M+1-\sum_{j=1}^{M}(j+1) \kappa_{j, i}$.

If $t=t_{0}=0$ is a critical value of $P$ then $P$ has repeated roots. If $P(z)=0$ has $k_{j, 0}$ roots of multiplicity $j+1$, the critical value $t_{0}$ is attained at $k_{j, 0}$ critical points of multiplicity $j$ each. Notice that if $P$ has a critical point of multiplicity $j$ at $z=0$, then $k_{j, 0}$ is odd, otherwise $k_{j, 0}$ is even. So to the zero level we associate the partition

$$
W_{0}=1^{k_{0,0}} 2^{k_{1,0}} \cdots(2 M+1)^{k_{2 M, 0}},
$$

$k_{0,0}=2 M+1-\sum_{j=1}^{M}(j+1) k_{j, 0}$.

Rem ark 8. If $P$ does not have a critical value at 0 then the partition associated to the zero level is $W_{0}=1^{2 M+1}$.

Definition 4.2. An odd polynomial $P$ belongs a stratum $\Sigma_{\mathbf{W}}$ with associated passport

$$
\mathbf{W}=\left\{W_{0}, W_{ \pm 1}, \ldots, W_{ \pm(c-1)}\right\},
$$

where $W_{0}$ is the partition of $2 M+1$ associated to the zero level and $W_{ \pm i}, i \neq 0$, is the partition of $2 M+1$ associated to the critical level $t_{ \pm i}$, as follows

$$
\begin{aligned}
& W_{0}=1^{k_{0,0}} 2^{k_{1,0}} \cdots(2 M+1)^{k_{2 M, 0}}, \\
& W_{ \pm i}=1^{\kappa_{0, i}} 2^{\kappa_{1, i}} \cdots(M+1)^{\kappa_{M, i}}, \quad i \neq 0 .
\end{aligned}
$$

Remark 9. A generic odd polynomial of degree $2 M+1$ has $2 M$ distinct non-zero critical points $\pm z_{i}, i=1, \ldots, M$ and $2 M$ distinct non-zero critical values $P\left( \pm z_{i}\right)= \pm t_{i}$, $i=1, \ldots, M$. The passport associated to a generic odd polynomial would be of the form

$$
\mathbf{W}=\left\{W_{0}, W_{1}, \ldots, W_{M}, W_{-1}, \ldots, W_{-M}\right\},
$$

where $W_{i}=1^{2 M-1} 2^{1}, i \neq 0$, is the partition associated to the critical value $t_{i}$, and $W_{0}=1^{2 M+1}$ is the partition associated to the non-critical zero level.

Definition 4.3. To the passport $\mathbf{W}$ we associate the secondary passport

$$
\mathbf{X}=\left\{X_{0}, X_{1}, \ldots, X_{c-1}\right\}
$$

where $X_{0}, \ldots, X_{c-1}$ are partitions of $2(2 M+1)$ and

$$
\begin{aligned}
& X_{0}=(2 \cdot 1)^{k_{0,0}}(2 \cdot 2)^{k_{1,0}} \cdots(2 \cdot(2 M+1))^{k_{2 M, 0}}, \\
& X_{i}=1^{k_{0, i}} 2^{k_{1, i}} \cdots(M+1)^{k_{M, i}}, \quad i=1, \ldots, c-1, \quad k_{j, i}=2 \kappa_{j, i} .
\end{aligned}
$$

We call the partition $X_{i}$ the secondary partition associated to $W_{i}$. The secondary passport associated to $\mathbf{W}$ is the passport of the even polynomial of degree $4 M+2$ which is the square of the original odd polynomial $P$. 
4.2. Main Theorem for odd polynomials. Consider the passport

$$
\mathbf{W}=\left\{W_{0}, W_{ \pm 1}, \ldots, W_{ \pm(c-1)}\right\},
$$

where the partitions $W_{i}$ of $2 M+1$ are of the form

$$
\begin{aligned}
& W_{0}=1^{k_{0,0}} 2^{k_{1,0}} \cdots(2 M+1)^{k_{2 M, 0}}, \\
& W_{i}=1^{\kappa_{0, i}} 2^{\kappa_{1, i}} \cdots(M+1)^{\kappa_{M, i}}, \quad i \neq 0,
\end{aligned}
$$

where a maximum of one of the $k_{i, 0}$ is an odd integer.

To this passport we associate the secondary passport $\mathbf{X}$ (see Definition ??). This is the passport of a stratum in the family of even polynomials of degree $4 M+2$ and so $T(\mathbf{X}), T\left(X_{i}\right)$ and $Y_{i}$ are all partitions as defined in Sections 2, 3.1.

We denote by $c_{\text {Morse }}$ the number of even Morse partitions in the secondary passport, that is, those of the form $X_{i}=1^{4 M-2} 2^{2}$.

THEOREM 4.4. The multiplicity of the restriction $\left.L L\right|_{\mathbf{W}}$ of the $L L$ mapping to the stratum $\Sigma_{\mathbf{W}}$ in the family of odd polynomials, determined by the passport $\mathbf{W}$, is given by the formula

$$
\mu\left(\left.L L\right|_{\mathbf{w}}\right)=\frac{(2 M+1)^{c-1}}{(2 M)^{c_{\text {Morse }}}} \frac{\# \operatorname{Aut}(T(\mathbf{X}))}{\# \operatorname{Aut}(\mathbf{X})} \prod_{i=0}^{c-1} \frac{\# \operatorname{Aut}\left(T\left(X_{i}\right)\right)}{\# \operatorname{Aut}\left(Y_{i}\right)},
$$

where $\mathbf{X}$ is the secondary passport associated to $\mathbf{W}$.

4.3. Proof of Main Theorem for odd polynomials. Before we begin the proof we need to prove the following elementary lemma.

Lemma 4.5. Consider odd polynomials $P_{1}$ and $P_{2}$. Then

$$
P_{1} \sim_{T} P_{2} \Longleftrightarrow P_{1}^{2} \sim_{T} P_{2}^{2} .
$$

Proof. If $P_{1} \sim_{T} P_{2}$ then there exists a homeomorphism $h$ such that $P_{1}=P_{2} \circ h$. Squaring both sides of this equation gives $P_{1}^{2}=P_{2}^{2} \circ h$, i.e., $P_{1}^{2} \sim_{T} P_{2}^{2}$. Now if $P_{1}^{2} \sim_{T} P_{2}^{2}$ then either $P_{1} \sim_{T} P_{2}$ or $P_{1} \sim_{T}-P_{2}$. Consider the homeomorphism $h: z \rightarrow-z$. Then $P \circ h=P(-z)=-P(z)$, i.e., $P \sim_{T}-P$. So if $P_{1}^{2} \sim_{T} P_{2}^{2}$ then $P_{1} \sim_{T} P_{2}$.

Thus there is a one-to-one correspondence between the topological type of an odd polynomial and that of its square and hence the number of topological types of odd polynomials $P$ from the stratum $\Sigma_{\mathbf{W}}$ is equal to the number of topological types of even polynomials $Q$ which are the squares of these odd polynomials. So to prove the theorem it is enough to work out the structure of the stratum on which $Q$ lies and then apply Theorem ??.

Consider the square of $P(z)=z^{2 M+1}+p_{1} z^{2 M-1}+\ldots+p_{M} z$, that is,

$$
Q(z)=P^{2}(z)=z^{2(2 M+1)}+q_{1} z^{2(2 M)}+\ldots+q_{2 M} z^{2} .
$$

R e mark 10. Note that within the space of polynomials of the form (??), the square root of the polynomial $Q$ is uniquely defined.

Notice that $Q$ is an even polynomial. Thus to prove the theorem it is enough to consider the stratum $\Sigma_{\mathbf{X}}$ determined by the passport $\mathbf{X}$, where a generic polynomial $Q$ from this stratum is the square of an odd polynomial $P$ from $\Sigma_{\mathbf{X}}$, and apply Theorem ??. 
Remark 11. Note that $Q$ has critical points when $Q^{\prime}(z)=2 P(z) P^{\prime}(z)=0$. So these critical points are all the critical points and roots of $P$.

Let $t$ be a non-zero critical value of $P$, and let $t$ occur at $\kappa_{i}$ critical points of multiplicity $i$. This is equivalent to saying that the polynomials $P+t=0$ and $P-t=0$ each have $\kappa_{i}$ roots of multiplicity $i+1$.

As the critical points of $P$ are also critical points of $Q$ (see Remark ??) and $Q(z)=$ $P(z)^{2}$, then $t^{2}$ is a critical value of $Q$. Consider the equation $Q-t^{2}=(P-t)(P+t)=0$. From the above argument this has $2 \kappa_{i}=k_{i}$ roots of multiplicity $i+1$, that is, $t^{2}$ occurs at $k_{i}$ points of multiplicity $i$. So to any critical value $t=t_{j}, j \neq 0$, of $P$ we associate a partition of the form

$$
W_{j}=1^{\kappa_{0}} 2^{\kappa_{1}} \cdots(M+1)^{\kappa_{M}}
$$

and to the related critical value $t^{2}=t_{j}^{2}$ of $Q$ we associate the secondary partition

$$
X_{j}=1^{k_{0}} 2^{k_{1}} \ldots(M+1)^{k_{M}}, \quad k_{i}=2 \kappa_{i}, \quad i=0, \ldots, M .
$$

Now let us consider the case when $t=t_{0}$ is the zero critical value of $P$. Firstly we assume that $z=0$ is not a critical point. Let $t_{0}$ occur at $k_{i}$ critical points of multiplicity $i$, where $k_{i}$ is odd only if there is a critical point at the origin. (We can see that $k_{i}$ is generally even because, for $z \neq 0$, if $P(z)=0$ then we also have $-P(-z)=0$. Of course if $z=0$ then $P(0)=-P(0)$ and we have an odd number of points on the zero level.)

This is the same as saying that $P=0$ has $k_{i}$ roots of multiplicity $i+1$. Thus, $Q=P^{2}=0$ must have $k_{i}$ roots of multiplicity $2(i+1)$, that is, the zero critical level occurs at $k_{i}$ critical points of multiplicity $2 i+1$.

So to a zero critical value $t=t_{0}$ of $P$ we associate the partition

$$
W_{0}=1^{k_{0}} 2^{k_{1}} \cdots(M+1)^{k_{M}},
$$

and to the zero critical value of $Q$ we associate the secondary partition

$$
X_{0}=2^{k_{0}} 4^{k_{1}} \cdots(2 M+2)^{k_{M}} .
$$

Remark 12. If the zero level is generic then $X_{0}=1^{4 M+2}$.

Thus to the stratum defined by the passport

$$
\mathbf{W}=\left\{W_{0}, \ldots, W_{ \pm(c-1)}\right\}
$$

where the $W_{i}$ are as defined above, we associate the stratum defined by the secondary passport

$$
\mathbf{X}=\left\{X_{0}, \ldots, X_{c-1}\right\}
$$

where

$$
\begin{aligned}
X_{0} & =(2 \cdot 1)^{k_{0,0}}(2 \cdot 2)^{k_{1,0}} \cdots(2 \cdot(M+1))^{k_{M, 0}}, \\
X_{i} & =1^{k_{0, i}} 2^{k_{1, i}} \cdots(M+1)^{k_{M, i}}, \quad i=1, \ldots, c-1, \quad k_{j, i}=2 \kappa_{j, i} .
\end{aligned}
$$

This stratum lies in the family of even polynomials, so applying Theorem ?? to this stratum ends the proof. 
4.4. Odd cacti and odd polynomials. Consider the cactus determined by an odd polynomial, $P$. Assume that $P$ has critical values $t_{0}, t_{ \pm 1}, \ldots, t_{ \pm(c-1)}$. Form a star with black vertices $t_{i}, i=0, \pm 1, \ldots, \pm(c-1)$, and label these accordingly with the integers $i=0, \pm 1, \ldots, \pm(c-1)$.

Definition 4.6. A cactus is called anti-symmetric if on rotating the cactus through 180 degrees about its central vertex and changing the signs of all its black vertices, we obtain the original cactus.

If an odd polynomial $P$ is not degenerate at the origin then its cactus is antisymmetric. However, this is not true if there is a degeneracy at the origin. To regain the anti-symmetry in the degenerate case, and thus enable us to recognise any cactus as one determined by an odd polynomial, we use modified cacti with white vertices 0 .

Definition 4.7. An odd cactus of degree $2 M+1$ is an anti-symmetric modified cactus of degree $2 M+1$ with white vertex 0 whose passport $\mathbf{W}$ is that of an odd polynomial.

We state the following result without proof. The proof follows directly from the enumeration result for general cacti in [?] along with symmetry arguments.

THEOREM 4.8. The number of rooted odd cacti with passport $\mathbf{W}=\left\{W_{0}, \ldots, W_{ \pm(c-1)}\right\}$ and associated secondary passport $\mathbf{X}=\left\{X_{0}, \ldots, X_{c-1}\right\}$ is

$$
\frac{(2 M+1)^{c-1}}{(2 M)^{c_{\text {Morse }}}} \prod_{i=0}^{c-1} \frac{\# \operatorname{Aut}\left(T\left(X_{i}\right)\right)}{\# \operatorname{Aut}\left(Y_{i}\right)}
$$

EXAmple 4.9. Consider odd cacti of degree 7 with passport,

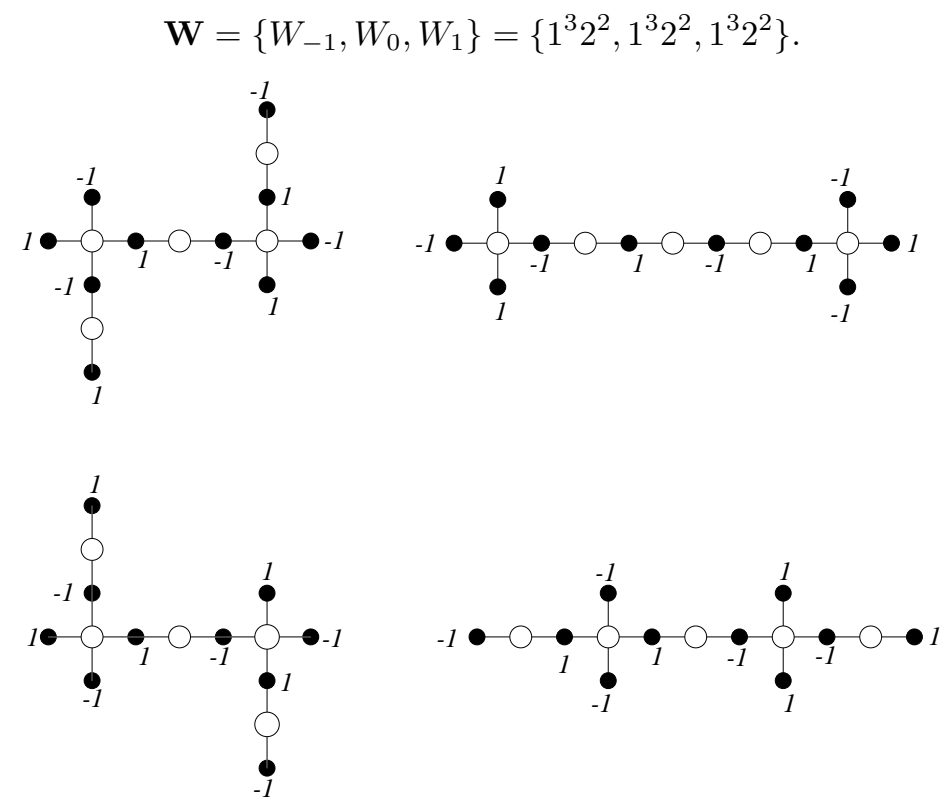

Figure 10. Odd cacti with passport $\mathbf{W}=\left\{1^{3} 2^{2}, 1^{3} 2^{2}, 1^{3} 2^{2}\right\}$ 
To this passport we associate the following values and partitions:

$M=7, c=2, c_{\text {Morse }}=0, X_{0}=2^{3} 4^{2}, X_{1}=1^{6} 2^{4}, Y_{0}=2^{1} 4^{1}, Y_{1}=1^{3} 2^{2}$, $T(\mathbf{X})=(9,4), T\left(X_{0}\right)=(9,2,2), T\left(X_{1}\right)=(4,1,2,2,2,2)$.

Using the formula from Theorem ?? we calculate the number of rooted odd cacti to be 28. In fact the only possible topological types of cactus with this passport are those in Fig. ??. There are 7 distinct rooted cacti of each topological type.

Now using Theorem ?? we calculate the multiplicity of the restriction of the $L L$ mapping to the stratum with passport $\mathbf{W}$ to also be 27.

\section{References}

[1] V. I. Arnol'd, S. M. Guseŭn-Zade, A. N. Varchenko, Singularities of Differentiable Maps II, Monogr. Math. 83, Birkhäuser, Boston, 1988.

[2] M. El Marraki, N. Hanusse, J. Zipperer and A. Zvonkin, Cacti, braids and complex polynomials, Sém. Lothar. Combin. 37 (1997), Art. B37b, http:/cartan.u-strasbg.fr/ slc

[3] V. V. Goryunov, S. K. Lando, On enumeration of meromorphic functions on the line, Preprint, 1997.

[4] I. P. Goulden, D. M. Jackson, Transitive factorisations into transpositions and holomorphic mappings on the sphere, Proc. Amer. Math. Soc. 125 (1997), 51-60.

[5] I. P. Goulden, D. M. Jackson, The combinatorial relationship between trees, cacti and certain connection coefficients for the symmetric group, European J. Combin. 13 (1992), 357-365.

[6] A. Hurwitz, Über Riemann'sche Fläche mit gegebenen Verzweigungspunkten, Math. Ann. 39 (1891), 1-66.

[7] A. G. Khovanskiǔ, S. Zdravkovska, Branched covers of $S^{2}$ and braid groups, J. Knot Theory Ramifications 5 (1996), 55-75.

[8] S. K. Lando, D. Zvonkine, On multiplicities of the Lyashko-Looijenga mapping on the discriminant strata, Preprint, 1997.

[9] E. Looijenga, The compliment of the bifurcation variety of a simple singularity, Invent. Math. 23 (1974), 105-116.

[10] V. Strehl, Reconstruction of a proof by A. Hurwitz, Preprint, 1996. 